\title{
Groupes sanguins et maladies de l'estomac
}

II existe fort peu de données sur les relations entre les groupes sanguins (determines pour garantir la sécurité de la transfusion) et les différentes maladies.

L'an dernier, une publication provenant de la Postgraduate Medical School de Londres (I. Aírd, H. H. Bentall et J. A. F. Roberts, Brit. Med. J. 1, 799, 1953) avait montré que les malades atteints de cancer de $\Gamma$ estomac appartenaíent au groupe A avec une frequence «significa-tivement» plus importante que celle des témoins: $46,19 \%$ au lieu de $41,38 \%$, et ceci aux dépens des groupes 0 et B.

Les mêmes auteurs ont étudié alors cette relation dans les cancers recto-côliques, bronchiques et mammaires, et sont arrives à des résul-tats négatifs: la repartition des groupes sanguins $0, \mathrm{~A}, \mathrm{~B}$ et $\mathrm{AB}$ est identique à celle des témoins; d'où la conclusion légitime que la localisation gastrique de la carcinomatose avait quelque chose à faire avec les groupes sanguins; et le désir de pousser cet ordre de recherche dans le domaine de $\Gamma$ affection organique la plus courante de 1'esto-mac: Yulcère.

Aides par J. N. Mehígan, les trois mêmes médecins viennent de publier (Brit. Med. J. 7 août 1954, p. 315) leurs nouvelles constata-tions concernant 3011 ulcéreux certains, provenant de 10 hôpitaux (dont 6 londoniens et 4 de province) et qui donnent ce résultat sur-prenant d'une augmentation de plus du síxième de la proportion du groupe 0 : (55,4\% au lieu de $47 \%$, alors que chacun des trois autres groupes voyait sa frequence relative diminuer d'autant). Cette distribution particulière concerne la maladie ulcéreuse, sans distinction de localisation gastrique ou duodénale.

Si les cancéreux gastriques font plus souvent partie du groupe A, les ulcéreux au contraire du groupe 0 , on peut en déduire qu'une personne appartenant au groupe $\mathrm{A}$ aura plus de probabilités de voir se développer un cancer d'estomac, tandis que les donneurs univer-sels (Gr. 0) auront une chance sur trois de plus que les porteurs d'autres groupes sanguins, de souffrir dun ulcère. L'explication de ces faits reste encore bien hypothétique. Ils con-firmeraient partiellement ce qu'on sait du facteur hérédítaíre dans la maladie ulcéreuse, qui jouerait un role dans $1 / 3$ des cas au moins, et 